Session IIIS

\title{
ONLINE ANALYSIS OF A RANDOM SIGNAL USING COMPUTER AIDED SYSTEM
}

\author{
Jerry K. Keska \\ College of Engineering, \\ University of Louisiana at Lafayette \\ P. O. Box-42972, Lafayette, Louisiana, USA 70506 \\ Raghavender Charupalli \\ Computer Science Department \\ University of Louisiana at Lafayette
}

\begin{abstract}
This paper attempts to describe an effective experimental approach to determine the characteristics of the random signal detected in the two-phase flow. This is a Computer-aided data acquisition system reporting data acquisition and data logging of parameters like root mean square (RMS), time, power spectral density (PSD), histogram and probability distribution of a random signal generated by two-phase flow in a vertical channel. The system employs virtual instrumentation software LabVIEW and data acquisition board (DAQ). The measurement and control are accomplished and integrated into the process by using a computerized data acquisition system (DAQ) and comprehensive virtual instrument (VI), which was developed using the LabVIEW application software and DAQ board. In addition, this system allows for easy modification and enhancement of a virtual instrument by modification of the software program.
\end{abstract}

\section{Introduction}

The combination of various parameters creates challenges in the description and determination of two-phase flow phenomena. The result is significant differences in reported results and difficulties in defining the experimental conditions, verification and validation of data, and a comparison of results. A combination of these independent variables and their distributions are creating phenomena like flow patterns, which cannot yet be objectively measured and incorporated into a phenomenological model due to its random character ${ }^{1,2,3}$. 
A novel research approach is being developed to characterize the random signal generated by two-phase air-water flow in a vertical pipe measuring $35 \mathrm{~mm}$ in diameter. In this paper emphasis is given to the analysis of a random signal obtained from two-phase flow as well as its characterization and computerized data acquisition using LabVIEW software. The data acquisition and analysis are accomplished and integrated by using a computerized data acquisition system and a comprehensive VI tool, developed using the LabVIEW application software. This system, which consists of the software and hardware, allows the modification and enhancement of the implemented procedures by application of the software program due to its overall versatility as an engineering tool.

The designed computerized measurement and data acquisition system will accomplish the following objectives ${ }^{7}$ :

- Acquire measured analog signals with high speed and accuracy.

- Interactively process and analyze measured data for immediate use and for future postprocessing.

- Generate necessary parameters for characterization of random signal.

- Interactively display the measured and analyzed data in graphical and/or numerical forms.

This paper reports the results of the development and application of VI for experimental analysis and documentation of results on the two-phase flow.

\section{Background}

In recent years, researchers are carrying out extensive work to satisfy the need for more reliable analysis and application of the two-phase flow detection systems. The most challenging, yet not well-defined problem is the definition of flow pattern and its characteristics. So one of the possibilities to describe two-phase flow in a channel is to characterize it as a random signal consisting of random fluctuations ${ }^{1,2,3}$. The fluctuations detected in the two-phase flow represent no deterministic fluctuations, which can be described by its frequency spectrum, amplitude statistical properties and time characteristics. To properly measure the different classes of signals, different averaging techniques and statistical methods are used. In particular, when measuring random signals the accuracy depends both on the measurement bandwidth and the measuring time. Measurement of a random signal's RMS value, histograms, and frequency spectrum is an effective attempt for characterizing such signals. In general, the measurement of fluctuations in frequency domain has received such great attention for so many years that it is surprising that the concept of determination of fluctuations does not have a universally accepted definition. At least part of the reason has been that some uses are most readily described in the frequency domain and other uses in the time domain; as well as in combinations of the two.

Proceedings of the 2003 ASEE Gulf-Southwest Annual Conference

The University of Texas at Arlington

Copyright (C) 2003, American Society for Engineering Education 
In a two-phase flow process, one can obtain its fluctuations in different domains by such parameters as power spectral density (PSD), RMS value and PDF, but one should not then expect an accurate prediction of it for any new condition. There is no existing model today that adequately describes the two-phase flow process. It is obvious that one cannot guarantee that this approach adequately describes the whole process, but the authors do feel that they are adequate for the description of a flow process phenomena that is presently encountered. Given a set of data of the frequency or time fluctuations, it is useful to characterize these fluctuations with reasonable and tractable models of performance. In the two-phase flow experiments, it is necessary to consider the fluctuations as random. As a tool to carry out the procedure, a computer-aided system (CAS) presented in Figure 1 and 3 is developed, which consists of the following components.

1.AT-MIO-16E-2 data acquisition (DAQ) board (E series architecture 500kSamples/s, 12-bit, 16 analog input multifunction DAQ, 16 single-ended/8 differential channels, two 12-bit analog outputs, 8 digital I/O channels).

2. CB-68LP I/O Connector, having dimension 7.62 by $16.19 \mathrm{~cm}$ equipped with 68 screw terminals for easily connecting 68-pin DAQ devices, has a vertical mounted 68-pin connector, which includes one 68-pin male SCSI connector for direct connection to 68pin cables.

3. LabVIEW professional development system. It is an essential tool for developing a computerized data acquisition system in order to integrate high accuracy measurements, data acquisition, interactive data processing and analysis for the feedback control ${ }^{5,9}$. This is used for data analysis and characterization of various parameters of a random signal thus obtained from random fluctuations of a two-phase flow that are accomplished and integrated by designing and implementing a computerized data acquisition system and comprehensive virtual instrument, developed using the LabVIEW application software ${ }^{8}$.

4. Two-phase flow system with sensors, measured systems and CCD camera.

\section{Personal Computer with software}

\section{Experimental System}

The experimental system shown in Fig. 2 and 3 consists of a vertical channel measuring $35 \mathrm{~mm}$ in diameter and 1 meter in length, which is positioned next to an air and water source. The bottom of the channel is provided with a mixing chamber, which allows the air to enter into the channel and mix fully with the water in the channel before entering the test section. The air input pressure to the mixing chamber ranges from zero to $600 \mathrm{KPa}$ and the airflow rate ranges from zero to 0.142 ( $\mathrm{std} \mathrm{m} 3 / \mathrm{min}$ ). The flow of air and water is controlled and measured by rotameters.

A separator at the top of the channel helps to release the air to the atmosphere and returns the water to the system supporting the closed-loop water system that is depicted in Figure 2.

Proceedings of the 2003 ASEE Gulf-Southwest Annual Conference

The University of Texas at Arlington

Copyright (C) 2003, American Society for Engineering Education 


\section{Camera}

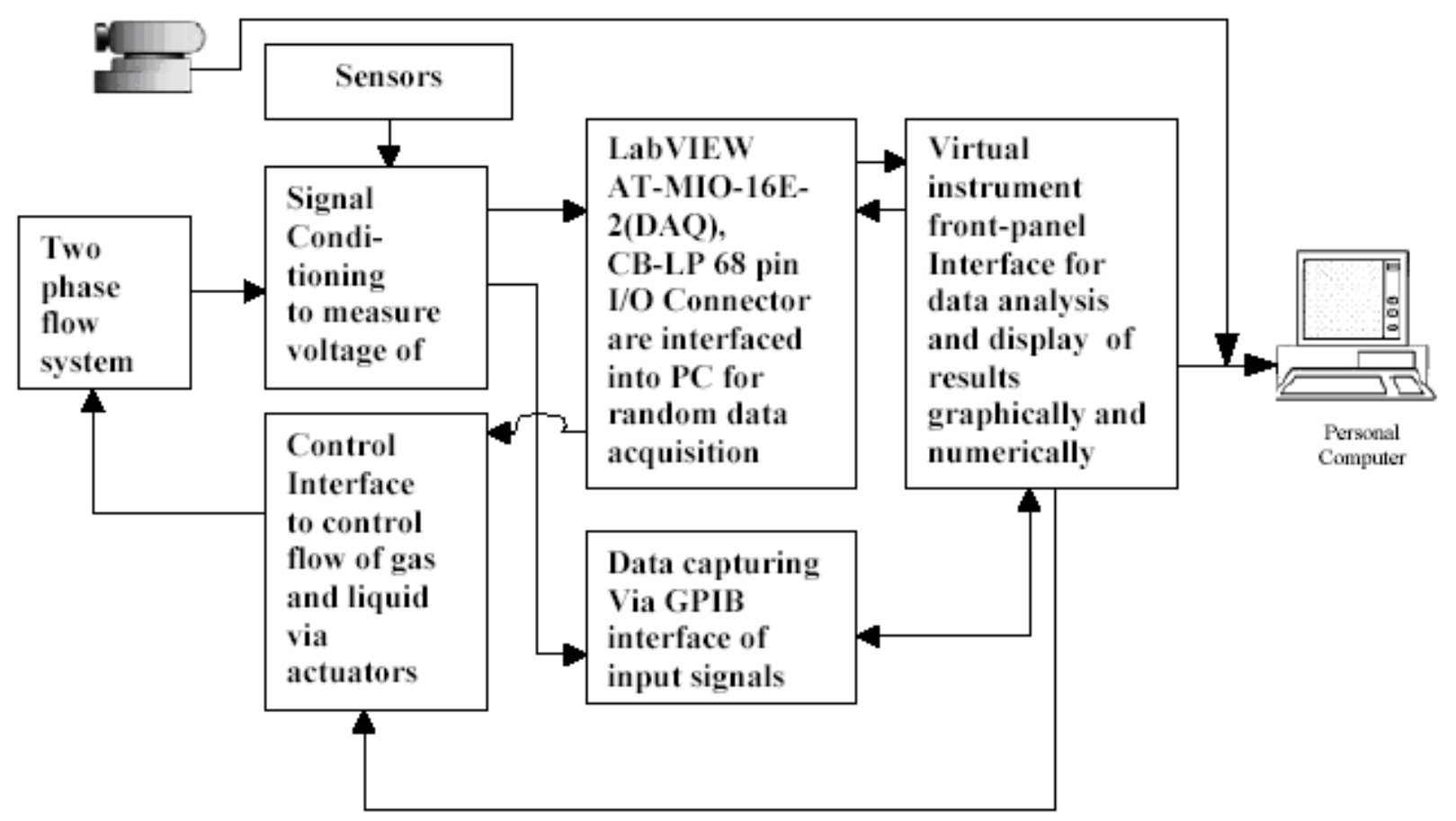

Figure 1. The general block diagram of a computer-aided system.

The two-phase flow parameters are measured using conductive and capacitive sensors, which are interfaced to the DAQ with a signal conditioner, generating signals proportional to the insitu concentration $^{1,2}$. A CCD camera generates real images of the two-phase flow in the system and is interfaced to the CAS. The random fluctuations of the two-phase flow air-water mixture is converted into digital signal and then interfaced to the PC employing a DAQ board, which is interfaced through the LabVIEW virtual instrument and displayed in the front panel. Then using LabVIEW VI the signal's various properties are determined. The properties considered for the random signal generated by the two-phase flow are RMSvalue, PSD, and PDF prominently, which are normalized, calibrated $(\mathrm{N} \& \mathrm{C})$ and displayed in the front panel of LabVIEW. Simultaneously the output signal is also normalized, scaled, displayed and sent to actuators to control the two-phase flow process. 


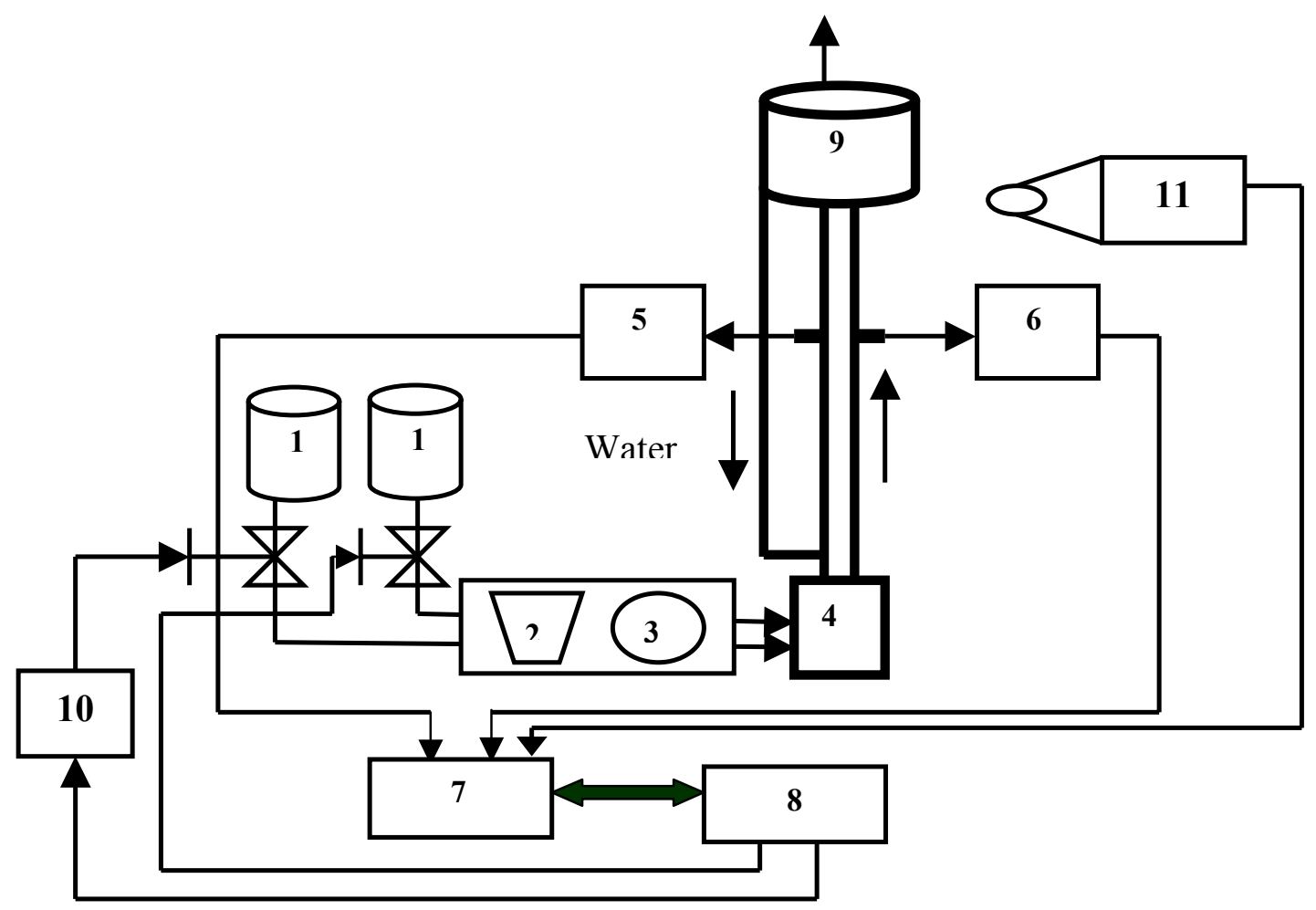

Figure 2. Two-Phase flow system

1. Tanks

2. Rotameters

3. Pressure gauge

4. Vertical channel

5. Conductive sensor

6. Capacitive sensor
7. Signal conditioner

8. CAS (Computer Aided

System)

9. Separator.

10. Flow Control System

11.Camera (CCD)

Based on the in-situ measurements, the control process is accomplished and integrated by using a computerized data acquisition system and a comprehensive "virtual instrument". 


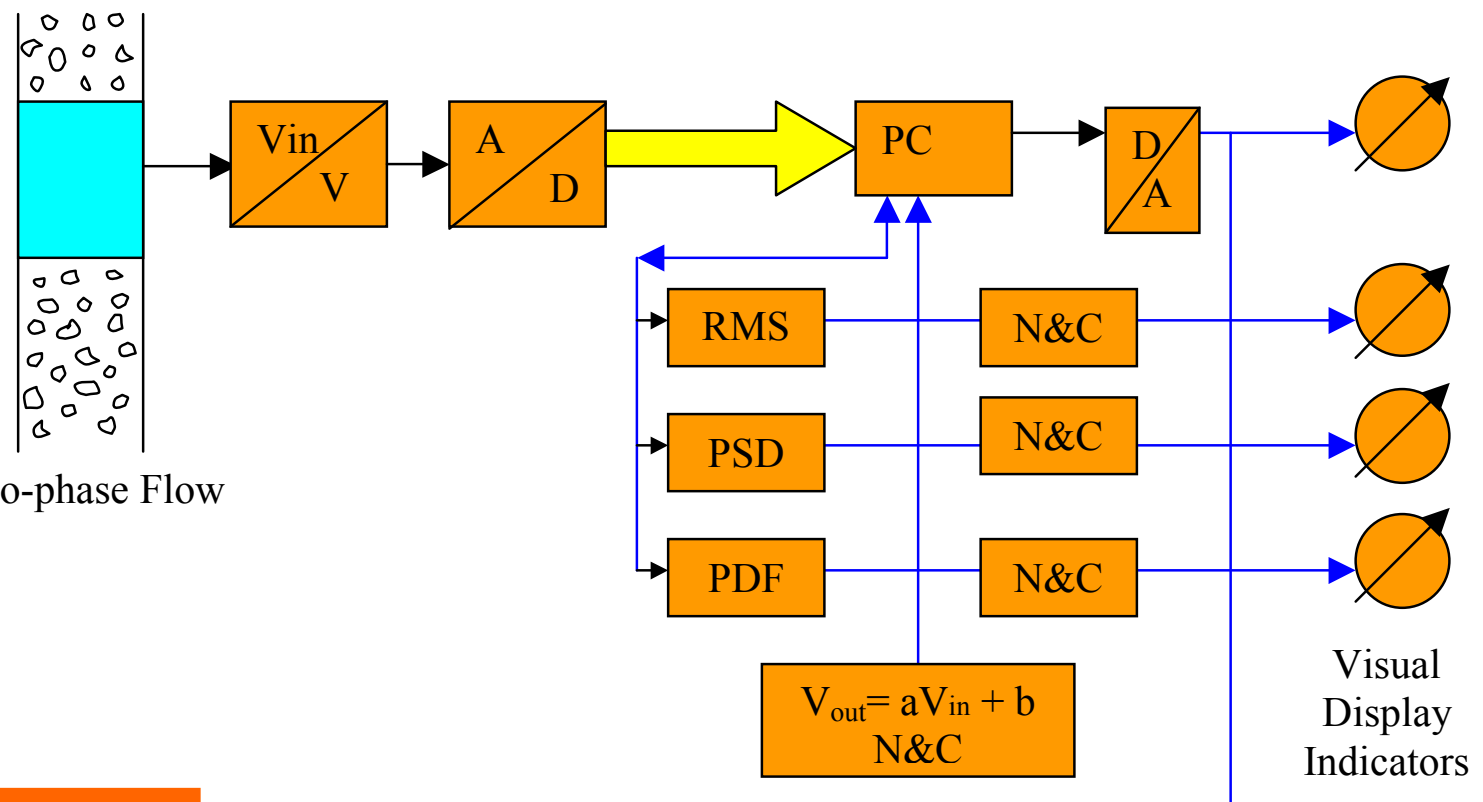

\section{Actuator}

Figure 3. Concept of a computer-aided control system for a random signal analysis.

\section{LabVIEW Data Acquisition and Analysis of a Random Signal}

Data acquisition is the digitizing and processing of a multiple sensor or signal inputs for the purpose of monitoring, analyzing and/or controlling systems and processes. The instantaneous random fluctuations generated by the two-phase flow are logged into the PC through the data acquisition system interfaced to it. This is accomplished using the LabVIEW DAQ board, ATMIO-16E-2 (E Series architecture 500kSamples/s, 12-bit, 16 analog input multifunction DAQ, 16 single-ended/8 differential channels, two 12-bit analog outputs, and 8 digital I/O channels). 


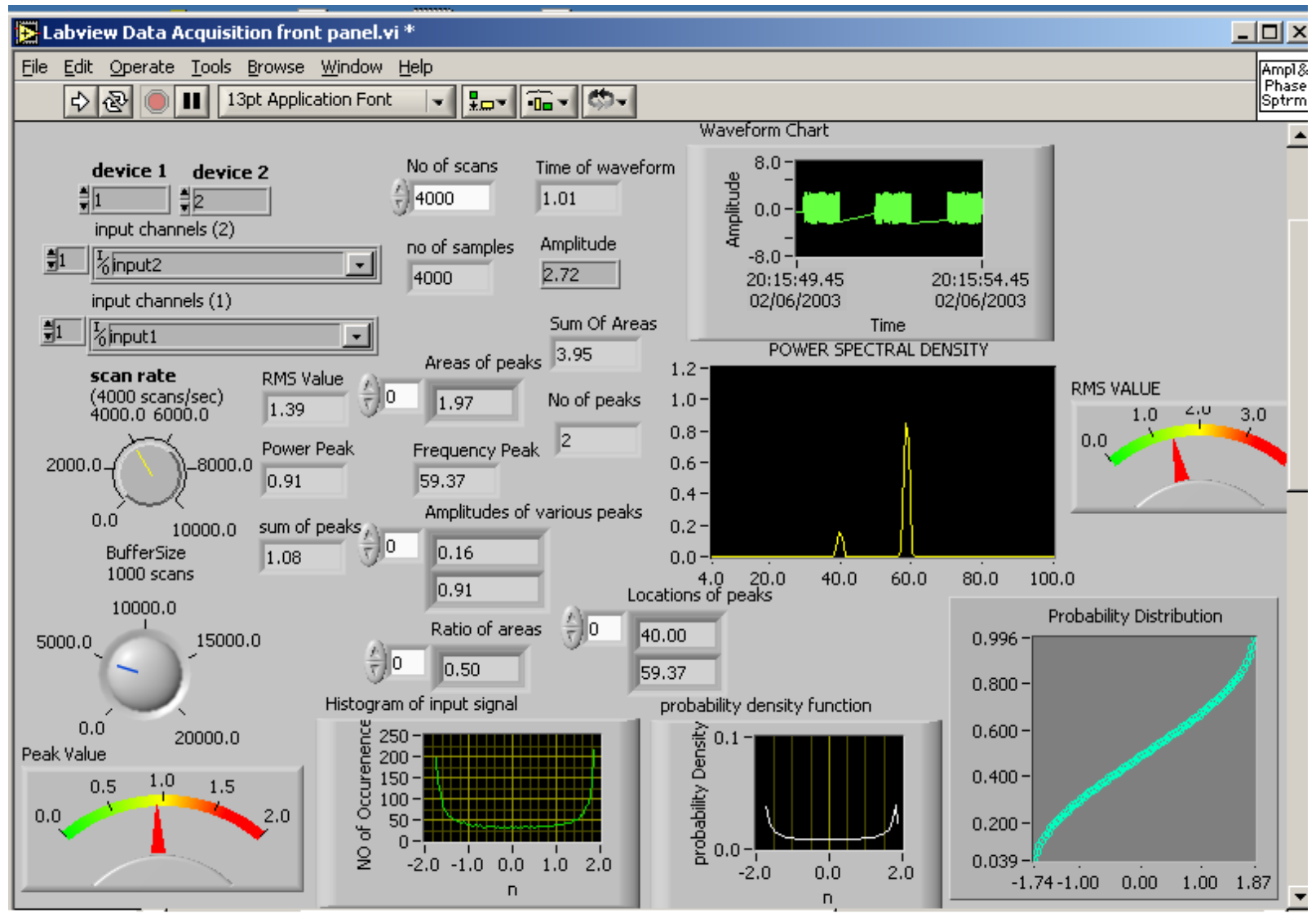

Figure 4. LabVIEW Virtual Instrument Front-Panel Interface

The I/O connector has 68 pins that can be connected to 68-pin accessories with the SH6868 cable or the ribbon, here ribbon is used. The logged random data is analyzed and characterized by the parameters such as RMSvalue, probability and frequency of occurrence functions, and parameters of peaks and PSD, which are displayed on the front panel of the LabVIEW.

A "virtual instrument "is developed, using the LabVIEW software application program. It integrates measurements, data acquisition, interactive data processing and analysis. The LabVIEW "virtual instrument's" front panel is given in Figure 3. It provides accurate and interactive control and display of measured and analyzed parameters such as RMSvalue of the signal, probability distribution function, PDF, histogram, peak detection, number of peaks its frequencies and amplitudes, and PSD. The measured and calculated parameters are displayed graphically, along with their values, and its limits wherever appropriate. The control of all functions and data acquisition settings is conveniently provided through the virtual instrument's "front panel" interface. The actual graphical block diagram to implement the data acquisition is shown in Fig.5. 


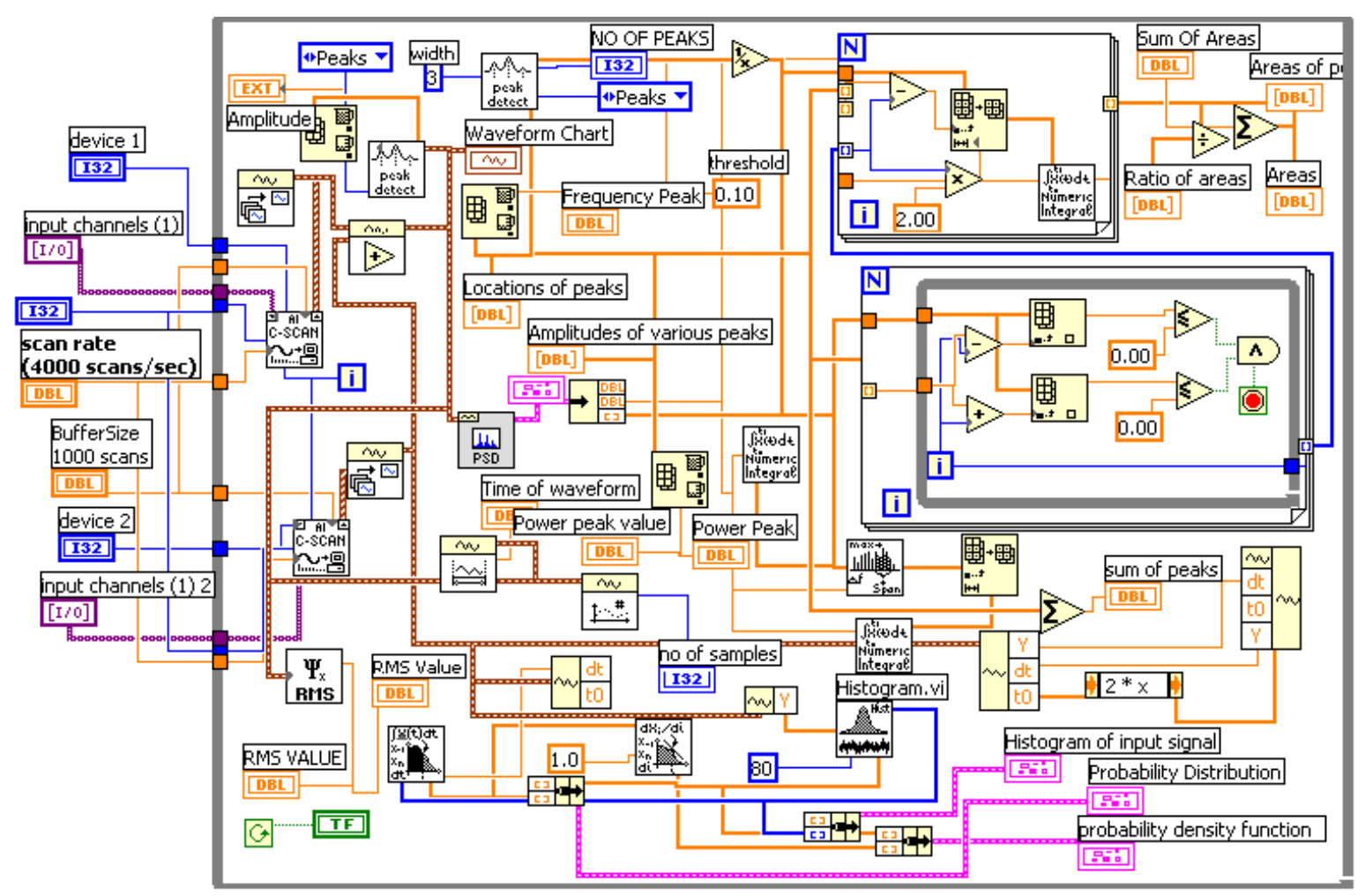

Figure 5. Block diagram of LabVIEW Data Acquisition System

\section{Results and Constraints}

The parameters considered for characterizing random signal such as RMSvalue, PSD, probability distribution function (PDF), number of peaks, area enclosed by the peaks, and histogram, are displayed on the front panel shown numerically and graphically. The peaks obtained for PSD are chosen in such a way that the peaks falling below certain threshold value $(0.1)$ be rejected while peaks above the threshold are only considered for display and records.

\begin{tabular}{|c|c|c|c|c|}
\hline $\begin{array}{c}\text { I/O Channel } \\
(1)\end{array}$ & I/O Channel (2) & $\begin{array}{c}\text { No of scans to } \\
\text { acquire (scans) }\end{array}$ & $\begin{array}{c}\text { Scan Rate } \\
(\text { scans/sec) }\end{array}$ & $\begin{array}{c}\text { Buffer Size } \\
\text { (scans) }\end{array}$ \\
\hline Input1 & Input2 & 4000 & 4000 & 5000 \\
\hline
\end{tabular}

Table 1. Control parameter of inputs

These inputs specified are depicted in Fig.4 and act as controls, which control and regulate the data acquisition. 
Input1 The DAQ I/O channel for this input is channel (1).

Input2 The DAQ I/O channel for this input is channel (2).

Number of scans to read is the number of scans the VI retrieves from the ongoing acquisition's buffer each time. This is equivalent to the number of points per channel. The number of scans to acquire is controlled by changing the value as needed which is shown in Fig.4 for proper working of VI number of scans which is taken as 4000 .

Scan rate is the number of scans per second acquired by the VI. This is equivalent to the sampling rate per channel. The number of scans to acquire per sec can also be varied as needed as shown in Fig.4 ranging from (0-10,000scans) and optimally 4000 is taken for the experiment.

Buffer size is the number of scans with input operations or updates with output operations the circular buffer holds. The buffer size first needs to be set to a certain value. If it is zero the virtual doesn't work and the buffer size ranges from $(0-20,000$ scans $)$ optimally 5000 scans have been taken.

The two physical waveforms obtained by LabVIEW DAQ are imposed in order to calibrate and test the system developed. And the properties of the imposed waveform is depicted in Table 2.The time of waveform is determined using waveform duration VI and similarly the RMS value is also determined with Basic (DC/RMS) VI.The signal is then sent to peak detection VI for determining the amplitude of waveform.

\begin{tabular}{|c|c|c|c|c|c|c|c|c|}
\hline $\begin{array}{c}\text { Time of } \\
\text { Waveform } \\
\text { (Sec) }\end{array}$ & $\begin{array}{c}\text { RMS } \\
\text { value }\end{array}$ & $\begin{array}{c}\text { Power } \\
\text { Peak } \\
\text { Value }\end{array}$ & $\begin{array}{c}\text { Areas } \\
\text { of } \\
\text { peaks }\end{array}$ & $\begin{array}{c}\text { No } \\
\text { of } \\
\text { peaks }\end{array}$ & $\begin{array}{c}\text { Sum } \\
\text { of } \\
\text { Peaks }\end{array}$ & $\begin{array}{c}\text { Amplitude } \\
\text { of various } \\
\text { peaks }\end{array}$ & $\begin{array}{c}\text { Locations } \\
\text { of peaks }\end{array}$ & $\begin{array}{c}\text { Frequency } \\
\text { Peak (Hz) }\end{array}$ \\
\hline 0.78 & 1.58 & 0.60 & 1.51 & 2 & 1.15 & 0.60 & 46.79 & 62.97 \\
\hline & & & 1.52 & & & 0.55 & 62.97 & \\
\hline
\end{tabular}

Table 2. Determined parameters of two physical signals imposed

Simultaneously the waveform is sent to determine the PSD of the signal, which computes the averaged PSD of the input signal. Each time waveform corresponds to a single FFT block and has to be passed individually to this VI. The power spectral density thus obtained is sent to the output cluster for displaying plotting spectral density with respective frequency values. The cluster is then unbundled and from that the array of magnitudes for power spectral density is sent to peak detectionVI, which outputs the amplitudes of various peaks, number of peaks, and locations of the frequency where the peaks occur. This is obtained by choosing the inputs the peak detector, namely threshold value (0.01) and width (5). 
The unbundled cluster is also wired to Array min max tool for determining the maximum value, which becomes the maximum power pea $\mathrm{k}$ of power spectral density. It is sent to Power and Frequency Estimate VI, which computes the estimated power and frequency around a peak in the power spectrum of a time-domain signal, which is displayed in the front panel.
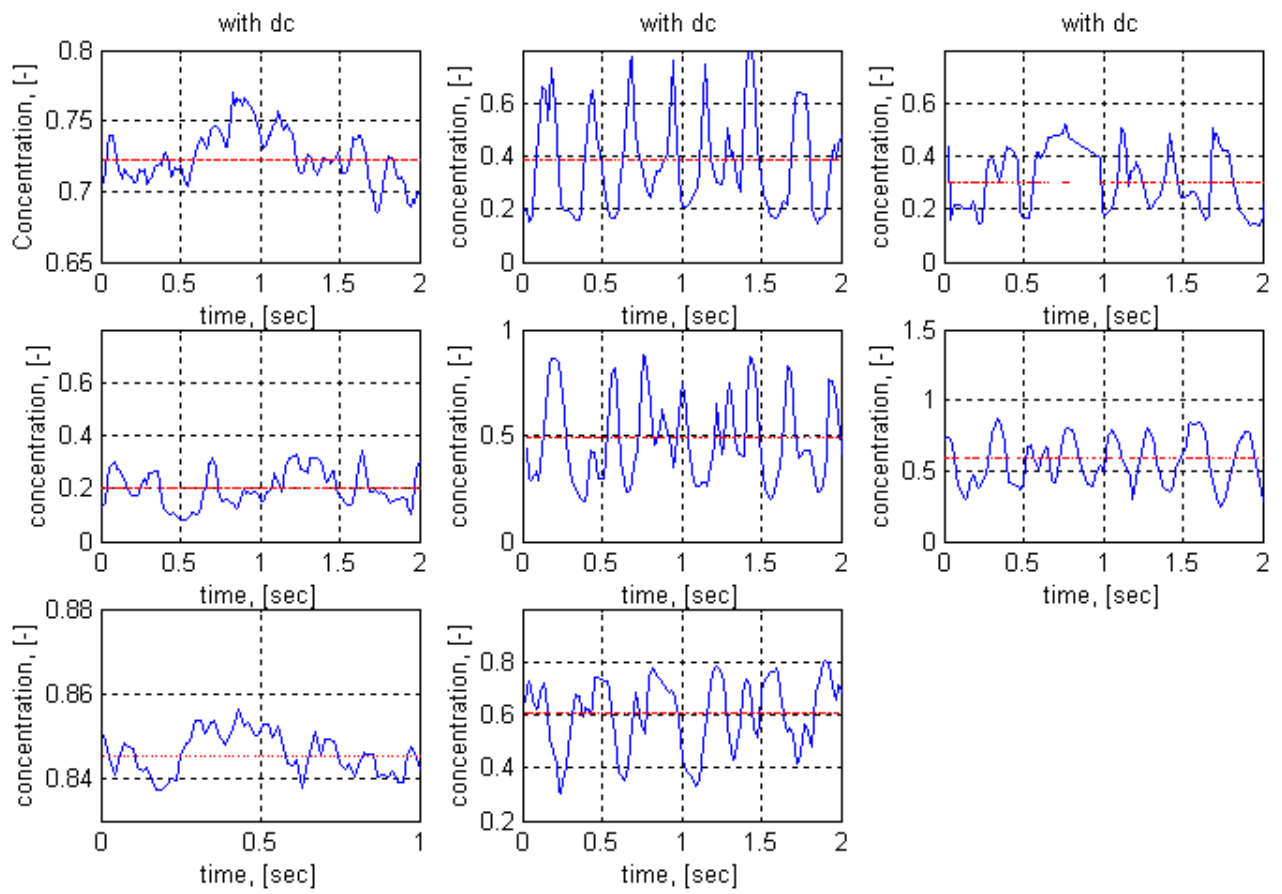

Figure 6. Set of two-phase flow concentration signals in time domain.

In above Fig. 6 each of the 8 concentration signals obtained from the two-phase channel in time domain is plotted and average values of the signal are given in Table 3 . The set of signals in time domain (Fig.6) are transferred into amplitude and frequency domains and displayed in Fig.7. CPD, PSD and CPSD functions. 

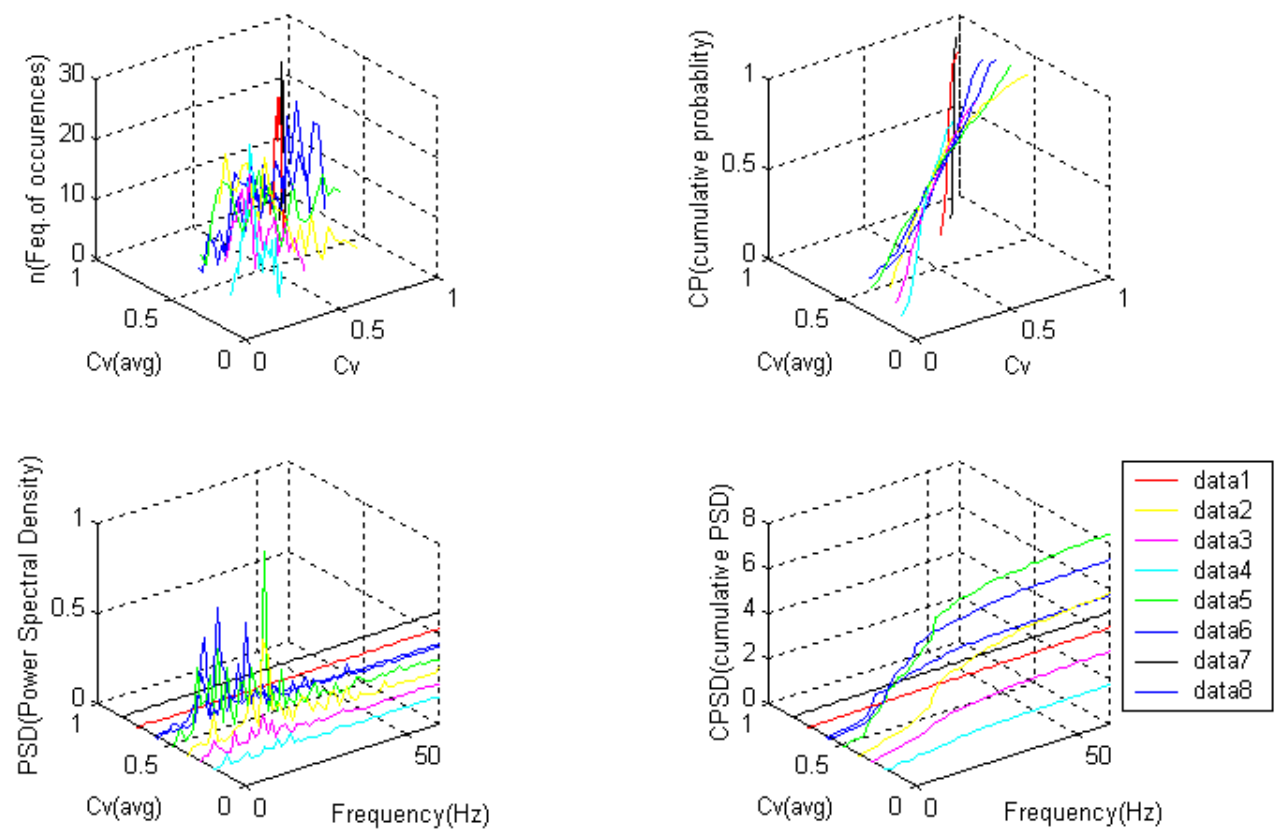

Figure 7. Experimental signals in amplitude and frequency domains.

\begin{tabular}{|c|c|}
\hline Signal & Cv (avg) \\
\hline data1 & 0.7223 \\
\hline data2 & 0.3838 \\
\hline data3 & 0.2984 \\
\hline data4 & 0.2046 \\
\hline data5 & 0.4895 \\
\hline data6 & 0.5843 \\
\hline data7 & 0.8453 \\
\hline data8 & 0.6069 \\
\hline
\end{tabular}

Table 3. Average concentration of two-phase flow in taken signals 


\section{Conclusion}

1. In the presented solution of an on-line analysis of a random signal, a two-phase flow system is used as a source of a random signal to determine the signal's various parameters in time, amplitude and frequency domains using a computer-aided data acquisition system. In this process a known deterministic signal is used for calibration and testing the system.

2. For characterization of the random signal the following functions are used: amplitude vs. time, PSD, PDF, RMS value, Histogram and the PSD peak value, number of peaks, its amplitudes and values of frequencies, and area of peaks. They are determined and interactively displayed in graphical and numerical form.

\section{References}

1. Keska, J. K. and B. E. Williams, "Experimental Comparison of Flow Pattern Detection Techniques for Air-Water Mixture Flow," International Journal of Experimental Heat Transfer, Thermodynamics, and Fluid Mechanics, Vol. 19, pp. 1-12, 1999.

2. Keska, J. K., M.D. Smith, and B. E. Williams, "Comparison Study of a Cluster of Four Dynamic Flow Pattern Detection Techniques," Flow Measurement and Instrumentation, Vol. 10, pp. 65-77, 1999.

3. Keska, J. K. and R. D. Fernando, "Average Physical Parameters in an Air-Water Two-Phase Flow in a Small Square-Sectioned Channel," Journal of Fluids Engineering, Vol. 116, pp. 247-254, 1994.

4. J.A. Barnes, et. al., "Characterization of Frequency Stability”, IEEE Trans. Instrum. Meas., Vol. IM-20, No. 2, pp. 105-120, May 1971.

5. Jeffrey Y. Beyon, National Instruments, LabVIEW Programming, Data Acquisition And Analysis, New Jersey, PHPTR, 1997.

6. N.Ertugrul, "Towards Virtual Laboratories: A Survey of LabVIEW-Based Teaching/Learning Tools and Future Trends", International Journal of Engineering Education, No. 16, Vol.3, 2000.

7. Kostic, M.,"Instrumentation with Computerized Data Acquisition for an Innovative Thermal Conductivity Apparatus," Conference Proceedings ASEE 1997 Annual Conference of American Society for Engineering Education, p.131-136.

8. C.V.Knight, et.al. "Modernization of a Mechanical Engineering Laboratory Using Data Acquisition with LabVIEW", Proceedings of the 1998 ASEE Conference, Session 2266, available at http://www.asee.org.

9. LabVIEW Manual, National Instruments Corp., 1996, http://www.ni.com.

10. Jeffrey Travis, LabVIEW For Everyone, 2ndEdition, NewJersey, PHPTR, 1997.

Proceedings of the 2003 ASEE Gulf-Southwest Annual Conference

The University of Texas at Arlington

Copyright (C) 2003, American Society for Engineering Education 


\section{RAGHAVENDER CHARUPALLI}

Raghavender Charupalli currently pursuing masters at University of Louisiana at Lafayette in Computer Science. His research interests are in Computer-aided Data Acquisition Systems and Web related applications.

\section{JERRY K.KESKA}

Dr.Jerry K.Keska serves as an Associate Professor of Fluid Power and Mechanical Systems, College of Engineering at the University of Louisiana at Lafayette. His research interests are in the areas of Microelectromechanical Systems (MEMS), dynamics of complex heterogeneous mixtures (multiphase, slurries), tribology, microheatexchangers; computer-aided measurement systems of concentration, film thickness and viscosity; turbulence and flow pattern phenomena in mixtures, and analysis and validation of deterministic and random signals.

Proceedings of the 2003 ASEE Gulf-Southwest Annual Conference

The University of Texas at Arlington

Copyright (C) 2003, American Society for Engineering Education

Proceedings of the 2003 ASEE Gulf-Southwest Annual Conference

The University of Texas at Arlington

Copyright (C) 2003, American Society for Engineering Education 